\title{
VÍNCULOS ENTRE LA COOPERACIÓN PARA EL DESARROLLO Y LA EDUCACIÓN EN LOS PAÍSES EN DESARROLLO*
}

\author{
Recibido: 20 de marzo de 2016 • Aprobado: 31 de octubre de 2016 \\ DOI: $10.22395 /$ seec.v20n42a6 \\ José Lorenzo Martín Arnaiz ${ }^{* *}$
}

\section{RESUMEN}

El objetivo de este trabajo es revisar si existen evidencias de los efectos de la cooperación internacional para el desarrollo en los avances de la educación en las economías en desarrollo. El método empleado para alcanzar este objetivo fue el de realizar una revisión de la literatura académica más relevante sobre el tema. En primer lugar, se revisa la literatura en la que se investiga el efecto de la ayuda al desarrollo sobre variables relevantes para los países menos desarrollados, excluidas las variables educativas. A continuación, se revisa la literatura en la que se analiza el efecto sobre la educación (variables flujo y stock). Las evidencias encontradas en la literatura no son concluyentes sobre el impacto de la cooperación en la educación en los países en desarrollo, dado que se encuentran tanto evidencias que identifican un efecto positivo como las que plantean que los efectos han sido negativos.

\section{PALABRAS CLAVE}

Cooperación internacional; Ayuda educativa; análisis de la educación; financiación de la educación; vínculos del desarrollo.

\section{CLASIFICACIÓN JEL}

I21, I22, O11, O19, O57

\section{CONTENIDO}

Introducción; 1. Los vínculos entre la ayuda al desarrollo y variables relevantes para los países menos desarrollados; 2 . Los vínculos entre la ayuda educativa y los avances de la educación; 3 Conclusiones; Bibliografía.

Este artículo de revisión, elaborado en 2015, nace de la intención de llevar a cabo una revisión de la literatura que analiza los vínculos entre la Cooperación internacional y los avances logrados en la educación en los países en desarrollo.

** Licenciado en Ciencias Económicas, Universidad de Valladolid, Valladolid, España. Doctor en Economía Aplicada, Universidad de Valladolid, Valladolid, España. Profesor colaborador fijo, Departamento de Economía Aplicada, Universidad de Burgos, Burgos, España. Miembro del Grupo de Investigación ERESCHA. Dirección: Plaza Infanta Doña Elena s/n. 09001. Burgos, España. Teléfono: 947259386. Correo electrónico: jlmartin@ubu.es. 


\section{LINKS BETWEEN DEVELOPMENT COOPERATION AND EDUCATION IN DEVELOPING COUNTRIES}

\section{ABSTRACT}

The goal of the paper is to review existent evidences of international cooperation effects for developments related with educational advancements in developing economies. The used method was based on the most relevant academic research found related to this specific topic. Initially, the effect of development cooperation over relevant variables for least developed countries was analyzed, excluding educational variables. Then, the literature that researches effects over education is analyzed (stock and flow variables). Evidences found in literature are not conclusive about the impact that cooperation has in developing countries, given that evidences show positive and negative effects on the same time.

\section{KEY WORDS}

International cooperation; Educational support; education analysis; education financing; development links.

\section{JEL CLASSIFICATION}

I21, I22, O11, O19, O57

\section{CONTENT}

Introduction; 1. Links between development support and the most relevant variables in least developed countries; 2 . Links between educational support and advancements in education; 3. Conclusions; Bibliography.

\section{VÍNCULOS ENTREA COOPERAÇÃO PARA O DESENVOLVIMENTO EA EDUCAÇÃO NOS PAÍSES EM DESENVOLVIMENTO \\ RESUMO}

O objetivo deste trabalho é revisar se existem evidências dos efeitos da cooperação internacional para o desenvolvimento nos avances da educação nas economias em desenvolvimento. O método empregado para alcançar este objetivo foi de realizar uma revisão da literatura acadêmica mais relevante sobre o assunto. Em primeiro lugar, se revisa a literatura na que se investiga o efeito da ajuda ao desenvolvimento sobre variáveis relevantes para os países menos desenvolvidos, excluídas as variáveis educativas. A continuação, se revisa a literatura na qual se analisa o efeito sobre a educação (variáveis fluxo e stock). As evidências encontradas na literatura não são concluintes sobre o impacto da cooperação na educação nos países em desenvolvimento, dado que se encontram tanto evidências que identificam um efeito positivo como as que propõem que os efeitos foram negativos.

\section{PALAVRAS CHAVE}

Cooperação internacional; Ajuda educativa; análise da educação; financiamento da educação; vínculos do desenvolvimento.

CLASSIFICAÇÃO JEL

I21, I22, O11, O19, O57

\section{CONTEÚDO}

Introdução; 1. Os vínculos entre a ajuda ao desenvolvimento e variáveis relevantes para os países menos desenvolvidos; 2 . Os vínculos entre a ajuda educativa e os avances da educação;

3 Conclusões; Bibliografia. 


\section{INTRODUCCIÓN}

Desde mediados del siglo XX, la educación ha sido el centro de las prioridades de las políticas de desarrollo y cooperación internacional. En la constitución de la Organización de Naciones Unidas en 1945 los países firmantes expresaban su confianza en la igualdad de oportunidades de la educación para todos y en el libre intercambio de ideas y conocimiento. La educación ha sido vista como un derecho universal y como uno de los determinantes principales del crecimiento económico en el largo plazo.

Sin embargo, no fue hasta 1990 cuando tuvo lugar la primera Conferencia Mundial sobre Educación en Jomtien (Tailandia). En esa ocasión, se compartía la visión de que era preciso centrar los esfuerzos en cubrir las necesidades de aprendizaje básicas. Aquí, la comunidad internacional se comprometió a alcanzar la Educación Primaria Universal (EPU) antes del fin del milenio. Diez años después, en Senegal el Foro de Dakar subraya que, para muchos países, la EPU estaba todavía lejos de ser una realidad. Durante este Foro se diseñó un marco de acción para la educación y se establecieron nuevos compromisos por la comunidad internacional para fortalecer la financiación internacional de la Educación Básica, para mejorar la previsibilidad de los flujos de ayuda, para asegurar una coordinación más efectiva entre los donantes y para desarrollar una aproximación sectorial real. En respuesta a ello, los países en desarrollo se comprometieron a poner en marcha planes de Educación Para Todos (EPT) y adoptar las medidas necesarias para alcanzar las metas de Dakar.

Los principales objetivos a alcanzar antes de 2015 eran la Educación Primaria Universal y la eliminación de las disparidades de género', metas que serían reforzadas unos pocos meses después por los Objetivos de Desarrollo del Milenio (ODM)². Para apoyar a los países en riesgo de no alcanzar estas metas se estableció en 2002 la Iniciativa de Seguimiento Rápido (ISR) por 22 donantes bilaterales y multilaterales. Consiste en una asociación global para acelerar la consecución de la Educación Primaria Universal en 2015, para proporcionar un mayor respaldo a la Educación Primaria y para mejorar su efectividad ${ }^{3}$. En un contexto más amplio, las declaraciones de las Conferencias de Monterrey (México) en 2002, Roma (Italia) en 2003, París (Francia) en 2005 y Bonn (Alemania) en 2009, también han tendido a reforzar los

1 Las otras metas establecidas en Dakar son el desarrollo de la educación infantil y la adquisición de las destrezas necesarias en la vida diaria, una mejora del 50 \% del nivel de la alfabetización adulta y una mejora en todos los aspectos de la calidad de la educación. En estas metas, sin embargo, se puso menos énfasis que en las disparidades de género y la EPU.

2 El objetivo N. 2 es alcanzar la Educación Primaria Universal para todos, y el objetivo N. ${ }^{\circ}$ 3, promover la igualdad de género y el empoderamiento de la mujer.

3 Desde septiembre de 2012, la Iniciativa de Seguimiento Rápido se ha convertido oficialmente en la Asociación Global para la Educación. 
compromisos respecto a los ODM y formalizar los esfuerzos de la comunidad internacional para la armonización y la efectividad de la ayuda. Asimismo, en la reunión del G8 de Gleneagles (Reino Unido) en 2005 se propuso el objetivo de duplicar la ayuda internacional y marcó la educación como un sector prioritario.

Si las estrategias de la comunidad internacional para respaldar los esfuerzos nacionales en educación han evolucionado a lo largo del tiempo, la Educación Básica permanece como un área prioritaria para la ayuda internacional. Sin embargo, hay pocas evidencias empíricas de la efectividad de la ayuda financiera en el nivel macroeconómico. El debate sobre la efectividad de la Ayuda Oficial al Desarrollo (AOD) se ha centrado en el impacto macroeconómico de la ayuda al desarrollo total. La ausencia de resultados consistentes y las muchas limitaciones metodológicas no resueltas han evitado el consenso en este tema particular. No obstante, la comunidad investigadora en los inicios del siglo XXI se ha orientado a tratar de salvar estas dificultades. Los investigadores se están moviendo de un modo gradual desde variables agregadas y cuestiones macroeconómicas amplias a centrarse en la efectividad de instrumentos específicos en objetivos definidos de una forma más estrecha.

Los estudios recientes sobre la efectividad de la ayuda para el comercio, ayuda para la salud y ayuda para la educación son parte de esta nueva tendencia. Los resultados obtenidos para los sectores sociales son variados, pero muestran que la ayuda tiende a mejorar la salud y la educación en los países en desarrollo. Sin embargo, los estudios relacionados con los sectores sociales son poco frecuentes $y$, desde una perspectiva metodológica, frágiles. Posibles explicaciones de la ausencia de evidencias empíricas sobre la efectividad de la ayuda sobre los avances de la educación se puede suponer que se derivan de los datos y de los métodos utilizados. Por lo que respecta a la ayuda para educación, la escasa fiabilidad de los datos ha sido el mayor impedimento para estudios serios sobre este tema. Así, aunque disponibles desde los años setenta, la utilización de datos sectoriales de ayuda solo es posible para el pasado reciente. El Instituto para las estadísticas de la UNESCO ha estado recogiendo datos fiables sobre diferentes indicadores relacionados con la educación desde 1999. El limitado período de tiempo y los problemas de calidad de los datos disponibles han limitado el campo de los economistas del desarrollo obligándolos a formular supuestos restrictivos, según señalan D’Aiglepierre y Wagner (2013).

El objetivo de este trabajo es determinar si existen vínculos entre la ayuda educativa y los avances de la educación en los países en desarrollo. La metodología empleada para lograrlo es llevar a cabo una revisión de la literatura académica que se ha centrado en el análisis de dicha relación. Se trata de trabajos que utilizan técnicas 
econométricas y modelos para realizar el análisis. Lo que se hace es presentar los estudios más novedosos, ya que nuestra intención es comprobar si los resultados en dichos estudios son tan poco concluyentes como los alcanzados en las investigaciones realizadas en la primera década del siglo XXI.

En un elevado número de estudios, la ayuda educativa tiene un impacto positivo y significativo en variables educativas flujo (tasa de matrícula), y stock (tasa de alfabetización y tasas de finalización). No obstante, también hay algunos estudios (menos numerosos) en los que dicho impacto es negativo y / o no significativo. Es decir, que los resultados en relación a dicho vínculo no son del todo concluyentes.

El artículo se estructura de la siguiente forma: en el epígrafe 1 se revisa la literatura en la que se investiga el efecto de la ayuda al desarrollo sobre variables relevantes para los países menos desarrollados, excluidas las variables stock de educación. En el epígrafe 2 se revisa la literatura en la que se analiza el efecto de la ayuda educativa sobre los avances de la educación en los países en desarrollo. Por último, en el epígrafe 3 se exponen las conclusiones que se extraen de la revisión de la literatura sobre los vínculos entre la ayuda educativa y las variables flujo y stock de educación que se ha llevado a cabo.

\section{LOS VÍNCULOS ENTRE LA AYUDA PARA EL DESARROLLO Y VARIABLES RELEVANTES PARA LOS PAÍSES MENOS DESARROLLADOS}

Diversos autores presentan modelos econométricos que relacionan de una manera estrecha la ayuda al desarrollo y otras variables como la renta, la tasa de mortalidad infantil, la tasa de fertilidad, la esperanza de vida, variables flujos de educación y variables stock de educación que actúan como regresores. Estas variables tienen una gran relevancia en los países en desarrollo, lo que suscita nuestro interés en la medida en que en dichos países no han logrado todavía el nivel alcanzado por los países desarrollados.

En primer lugar, Clemens, Radelet y Bhavnani (2004) analizan el impacto de diferentes categorías de ayuda en el crecimiento del producto interior bruto (PIB) per cápita real en los países en desarrollo mediante el uso de regresiones por mínimos cuadrados ordinarios en dos fases (2MCO). Detectan un importante impacto positivo de la ayuda educativa sobre el crecimiento económico en el largo plazo.

Por su parte, Asiedu y Nandwa (2007) examinan si la ayuda foránea desembolsada en educación tiene un efecto significativo sobre el crecimiento económico en 90 países en desarrollo en el período 1990-2004, a través de la aplicación del método generalizado de momentos (MGM) con un panel dinámico. Cabe destacar que 
el coeficiente estimado de la ayuda agregada no es significativo. Los resultados también sugieren que la ayuda en Educación Primaria estimula el crecimiento en los países de renta baja, pero la ayuda en educación pos-primaria no tiene un impacto significativo sobre el crecimiento. Para los países de renta media, la ayuda en Educación Primaria y Secundaria tiene un impacto negativo y significativo en el crecimiento, mientras que la ayuda en Educación Superior tiene un efecto positivo. Por tanto, es importante tener en cuenta la heterogeneidad de los países receptores cuando se analizan las relaciones ayuda-crecimiento.

Asimismo, Asiedu (2014) analiza si la ayuda foránea en educación tiene un efecto significativo sobre el crecimiento en 38 países de África Subsahariana en el período 1990-2004. Con este fin hace la estimación con el método MGM y con efectos fijos. Se encuentra que la ayuda en Educación Primaria tiene un efecto positivo y significativo sobre el crecimiento. Además, la ayuda en educación pos-primaria tiene un efecto adverso o no tiene un impacto significativo sobre el crecimiento. Por último, el crecimiento económico se incrementa al mismo tiempo que aumenta la ayuda en Educación Primaria como fracción de la ayuda educativa total.

Por otro lado, Neira y Portela (2003) intentan cuantificar el efecto de la ayuda educativa sobre la tasa de mortalidad infantil, la tasa de fecundidad y la esperanza de vida en 18 países latinoamericanos en el período 1980-2000. Para lograr su objetivo, realizan la estimación con efectos fijos. En concreto, el incremento de un dólar per cápita de la ayuda educativa ( 0,8 dólares de media) supondría un descenso del $6 \%$ en la tasa de mortalidad infantil, una reducción del $11 \%$ en la tasa de fecundidad (aunque desde un punto de vista estadístico no significativa) y un aumento de casi medio año en la esperanza de vida. Asimismo, se aprecia que el efecto de la ayuda educativa es de una magnitud mayor que el correspondiente a la ayuda total.

Se puede destacar también el trabajo de Arvin y Lew (2009) que, mediante el uso del método de Causalidad de Granger, investigan si los flujos de ayuda foránea influyen en la posterior escolarización, si las matrículas actuales influyen en la posterior recepción de la ayuda o si la causalidad actúa en ambas direcciones de un modo simultáneo. Al utilizar datos de ayuda per cápita en países en desarrollo en el período 1980-2002, los resultados sugieren que la relación ayuda-educación varía de una forma considerable a través de las submuestras definidas según la región geográfica de los países receptores. En algunos casos hay un vínculo positivo entre ayuda y educación y, en otros casos las variables no están relacionadas.

Asimismo, cabe reseñar el trabajo de Thiele, Nunnenkamp y Dreher (2007), que usan modelos de Tobit, de elección discreta (de probabilidad no lineal), para valorar si 
los donantes distribuyen la ayuda total en educación y la ayuda en educación básica (comprometida) en concordancia con los indicadores de necesidad relacionados con los objetivos de Desarrollo del Milenio (ODM) para una muestra de 140 países receptores. En las estimaciones de las regresiones ponderadas por la población, indicadores relacionados con los ODM en el campo de la educación (tasas de finalización de la Educación Primaria y el promedio de años de escolarización) afectan las decisiones de los donantes. Dichas regresiones sugieren una orientación de la ayuda acorde con los indicadores específicos de necesidad, incluso cuando ciertos indicadores como las tasas netas de matrícula en primaria y la relación alfabetización hombres / mujeres permanecen no significativos. En cualquier caso, para la educación, el vínculo entre los indicadores de necesidad y las categorías de ayuda permanece débil; solo el promedio de años de escolarización es significativo en las diversas especificaciones del modelo ponderado. En especial en Educación Primaria, hay una considerable brecha entre la retórica de los donantes y la asignación de la ayuda.

Kasuga (2008) investiga si los flujos de ayuda educativa a los países en desarrollo se ajustan bien a sus prioridades de desarrollo en el ámbito de la educación. Tras utilizar el modelo de Tobit, no encuentra evidencias de que las necesidades de educación afecten la ayuda en este sector, salvo en los países de renta media y alta de Asia Oriental. Los resultados muestran que la burocracia y la corrupción en los países receptores impiden una asignación eficiente.

Por otro lado, Dreher, Gehring y Klasen (2013) examinan si los donantes conceden más ayuda a los países con mayores brechas de género (las necesidades) en educación, salud, empleo o derechos de las mujeres, o más bien recompensan las mejoras en dichos indicadores (el mérito) en 95 países receptores, en el período 1973-2011, mediante la utilización del Método de Máxima Verosimilitud de Poisson (MMVP). Se encuentra alguna evidencia de que los donantes incrementan la ayuda a los países donde las necesidades en términos de brechas de género y bajos niveles de logro en indicadores de educación son mayores. Además, los donantes parecen recompensar a los países con una mayor representación femenina en los parlamentos con más ayuda. Estos efectos son más pronunciados entre los buenos donantes (Dinamarca, Holanda, Noruega Y Suecia) y los UE-3 (Francia, Alemania y Reino Unido), así como entre los donantes con una mayor representación femenina en el Gobierno o con ministras en el área de desarrollo. No se encuentra evidencia de que los donantes asignen la ayuda basándose en el mérito, en el sentido de recompensar a los países que logran reducciones en las brechas de género o reducir privaciones femeninas en educación. 
Por otra parte, Kleemann, Nunnenkamp y Thiele (2014) contrastan la hipótesis de que la desigualdad de género en educación es más probable que afecte la asignación de la ayuda de los países donantes con un liderazgo femenino en el ministerio relevante, a través del uso de modelos de elección discreta (de probabilidad no lineal) en el período 1995-2011 con datos de 23 donantes del Comité de Ayuda al Desarrollo (CAD) y 123 países receptores. No se encuentra evidencia de una asignación de la ayuda a la educación basada en las necesidades. Cuando se decide la selección de receptores, los líderes (tanto hombres como mujeres) del ministerio responsable de la cooperación al desarrollo parecen haber recompensado a los países con más años de educación de todos los jóvenes, o en concreto de mujeres. Los resultados para el importe de la ayuda dada a los receptores elegibles también apuntan a una asignación de la ayuda a la educación basada en el comportamiento, una vez que las necesidades generales, en términos de rentas per cápita promedio, están controladas. Mayores promedios de años de educación, con independencia de si se define de un modo neutral o específico de género, están asociados con una mayor ayuda a la educación concedida por los líderes (tanto hombres como mujeres) en todos los niveles de educación. Las estimaciones indican menores diferencias de género en el comportamiento de la asignación. Mientras las líderes femeninas recompensan los logros educativos en educación pos-secundaria de un modo más intenso que los líderes masculinos cuando seleccionan receptores de la ayuda a la educación a este nivel, los líderes masculinos recompensan los logros educativos en educación pos-secundaria de una forma más intensa cuando deciden sobre el importe de la ayuda para los receptores seleccionados.

Por su parte, Van de Sijpe (2010) analiza si el uso de la ayuda educativa foránea por el gobierno socio para financiar una intervención de desarrollo reemplaza la financiación interna dirigida a este mismo fin en 108 países en el período 1990 2004. En los modelos estáticos con efectos fijos la ayuda educativa muestra una correlación de casi uno por uno con el gasto público en educación del país receptor de la ayuda. Sin embargo, este efecto se convierte en impreciso y volátil en los modelos dinámicos (datos de panel) estimados con el sistema MGM. Por tanto, no se pueden extraer conclusiones firmes.

Por último, Ziesemer (2011) también examina el impacto de la AOD en el gasto público en educación en 35 países de renta baja en el período 1981-2005, mediante la aplicación del método de mínimos cuadrados ordinarios (MCO) con efectos fijos. Los resultados muestran que la AOD y las remesas de los trabajadores inducen al Gobierno a gastar más en educación. Por consiguiente, en las políticas de los países pobres de la muestra estos flujos monetarios son todos complementarios en lo que respecta a la educación. 


\section{VÍNCULOS ENTRE LA AYUDA EDUCATIVA Y LOS RESULTADOS EDUCATIVOS}

A continuación se presenta una serie de trabajos en los que se investiga el efecto de la ayuda dirigida al sector de la educación en los resultados educativos de países en desarrollo localizados en una región geográfica concreta. Es el caso de los estudios de Neira y Portela (2004), Moe (2008), Anwar y Aman (2010) y Yogo y Mallaye (2014). Estos trabajos utilizan técnicas econométricas y modelos para realizar el análisis.

Neira y Portela (2004) examinan el papel que la ayuda al desarrollo en el ámbito educativo ejerce sobre las tasas de analfabetismo en 12 países latinoamericanos, a través de la estimación de un panel de datos con efectos fijos. Cabe destacar la no significatividad de la variable ayuda educativa. Asimismo, encuentran una relación positiva entre ambas variables (el coeficiente de correlación es de 0,45).

Por su parte, Moe (2008) intenta investigar la relación entre la AOD en educación y el desarrollo humano y educativo en ocho países del sudeste asiático con un nivel medio de desarrollo humano en el período 1990-2004, mediante la aplicación del método de MCO. Se detecta que la AOD destinada a los niveles no especificados de la educación o a la educación pos-secundaria muestra una asociación significativa con el desarrollo humano, mientras que la AOD suministrada para la educación básica, la educación secundaria y la educación pos-secundaria tiene una significativa relación con el desarrollo educativo.

Por otro lado, Anwar y Aman (2010) examinan el impacto de la ayuda foránea desembolsada en el sector de la educación en la tasa de alfabetización de Pakistán. Tras usar el método de MCO, los resultados muestran que el coeficiente de la ayuda educativa es positivo y significativo, pero su valor es muy pequeño, lo que implica que una muy pequeña porción de la ayuda se utiliza de una forma efectiva y eficiente, mientras que la mayor parte de ella se desaprovecha. Tras utilizar el test de Cointegración de Johansen, los resultados muestran un positivo y significativo impacto de la ayuda foránea en el largo plazo. Por último, al emplear un Modelo de Corrección de error la variable de ayuda muestra una relación no significativa, lo que significa que no tiene un considerable impacto sobre la tasa de alfabetización en el corto plazo.

Por último, Yogo y Mallaye (2014) analizan la efectividad de la ayuda y su uso eficiente en el intento de alcanzar la Educación Primaria universal en 35 países de África Subsahariana en el período 2000-2010, mediante la aplicación de los métodos MCO con efectos fijos aleatorios y variables instrumentales, mínimos cuadrados generalizados (MCG), 2MCO y un modelo de Tobit. Los resultados sugieren que una mayor ayuda educativa incrementa de un modo notable la tasa de finalización 
en Educación Primaria. Este resultado es consistente con la utilización de varios métodos de estimación, la inclusión de instrumentos para tener en cuenta la endogeneidad de la ayuda y el conjunto de variables de control incluidas en las regresiones. Además, este trabajo muestra que hay una fuerte heterogeneidad en el uso eficiente de la ayuda, que está correlacionado de una forma positiva con el tamaño de la población urbana, el nivel de desarrollo, la calidad de la gobernanza y el nivel inicial de educación.

A continuación se presenta una serie de trabajos en los que se examina el impacto de la ayuda dirigida al sector de la educación en los resultados educativos de países en desarrollo de los cinco continentes. Es el caso de los estudios de Michaelowa (2004), Fielding, Mc. Gilllivray y Torres (2006), Wolf (2007), Michaelowa y Weber (2007a y 2007b), Dreher, Nunnenkamp y Thiele (2008), Gymah-Brempong y Asiedu (2008), Findley et al. (2009), Christensen, Homer y Nielson (2010 y 2011), Ziesemer (2011 y 2012), D’Aiglepierre y Wagner (2013), Birchler y Michaelowa (2013) y Maiga (2014). Estos trabajos utilizan técnicas econométricas y modelos para realizar el análisis.

Michaelowa (2004) analiza la efectividad de la ayuda en el sector de la educación en 80 países de renta baja y de renta media-baja, mediante la utilización de modelos estáticos con efectos fijos y aleatorios y un panel de datos dinámico, que estima con el Sistema MGM. Los resultados muestran que la ayuda educativa aumenta la tasa de matrícula en la enseñanza Primaria. Este resultado es bastante consistente para diferentes datos y métodos de estimación. Las estimaciones del coeficiente para la variable ayuda varían mucho entre los resultados más pesimistas y más optimistas. Según las estimaciones basadas en la cooperación técnica, un incremento del 1 \% en la tasa de matrícula Primaria requiere un incremento de 25 millones de dólares en la ayuda educativa o un 0,15 \% del PIB de un país receptor mediano. Además, bajo condiciones de un mal gobierno, el impacto de la ayuda sobre la escolarización se vuelve negativo.

Por otro lado Fielding, Mc. Gillivray y Torres (2006) examinan el impacto de la ayuda foránea en una serie de indicadores del desarrollo humano, entre los que se halla la educación, en 48 países, en el período 1995-2004, a través de la aplicación del método 3MCO. Estos autores distinguen entre el efecto directo de la ayuda, en promedio y efecto equilibrio, que es el efecto final de la ayuda sobre el desarrollo tras las interacciones entre los diferentes indicadores de desarrollo. El efecto directo de la ayuda sobre la educación es pequeño, pero positivo en promedio, y desde un punto de vista estadístico no significativo. Sin embargo, el efecto equilibrio de la ayuda sobre la variable educativa es casi siempre positivo. Hay una fuerte interacción positiva entre salud y educación. Asimismo, la educación responde de un modo positivo a las mejoras en los activos materiales y la mortalidad infantil. 
Por su parte, Wolf (2007) analiza los efectos del volumen y de la volatilidad de la ayuda en los avances en educación en 52 países en desarrollo en el período 19802002, mediante el uso de un modelo de ecuaciones simultáneas y el método MCO. La fracción de la AOD para la Educación Primaria es positiva y significativa en dos especificaciones, lo que implica que la ayuda educativa tiene un impacto positivo tanto en la tasa de finalización en Educación Primaria como en la tasa de alfabetización de los jóvenes. El coeficiente de la ayuda como fracción de la Renta Nacional Bruta (RNB) es negativo y significativo en tres casos. El coeficiente para la volatilidad de la ayuda es negativo y significativo para la tasa de finalización de la Educación Primaria, lo que significa que una mayor volatilidad de la ayuda puede reducir la eficiencia de la educación. Sin embargo, el coeficiente de la volatilidad de la ayuda es positivo y significativo para la tasa de alfabetización de los jóvenes.

Asimismo, Michaelowa y Weber (2007a) examinan la efectividad de la ayuda en el sector de la educación en 120 países de renta baja y renta media-baja, a través de la utilización de paneles de datos estructurales y anuales, que estiman con el sistema MGM. A pesar de algunas diferencias en los resultados basadas en datos y métodos de estimación diferentes, la aplicación empírica al sector educativo permite concluir que la ayuda educativa aumenta las tasas de matrícula y finalización en la enseñanza Primaria. Por consiguiente, se observa un efecto positivo de la ayuda, aunque los coeficientes estimados del impacto de la ayuda son bastante pequeños. En el caso más optimista, un incremento de la ayuda del 200 \% conduciría a un aumento de la matrícula neta primaria de 2,5 puntos porcentuales. Solo donde la ayuda educativa es baja en términos relativos al principio, puede haber al inicio un mayor impacto. Se detectan rendimientos decrecientes de la ayuda educativa. Además, bajo condiciones de un mal gobierno, el impacto de la ayuda sobre la escolarización se vuelve negativo.

En otro trabajo del mismo año, Michaelowa y Weber (2007b) investigan el impacto de la ayuda educativa desagregada en los avances en Educación Primaria, Secundaria y Terciaria en 100 países de renta baja y renta media-baja, mediante el uso de modelos estáticos con efectos fijos y aleatorios, y un panel de datos dinámico, que estiman con el sistema MGM. Se encuentran evidencias de un efecto positivo de la ayuda educativa en los tres niveles de enseñanza. Sin embargo, los efectos estimados son bastante pequeños. Según los resultados de la estimación más optimista, el incremento de la ayuda en cualquier nivel de educación del 1 \% del PIB del país receptor mejorará las tasas de finalización un máximo de 2,5 puntos porcentuales. Aunque los resultados pueden subestimar el efecto verdadero de la ayuda debido a problemas de precisión de datos en este nivel de desagregación, confirman de una manera aproximada los resultados de estudios anteriores basados en la ayuda total a la educación. Asimismo, 
en línea con dichos estudios, para la enseñanza Primaria y Secundaria, se encuentran evidencias de rendimientos decrecientes de la ayuda, así como de una interdependencia de la efectividad de la ayuda y el buen gobierno.

Por su parte, Dreher, Nunnenkamp y Thiele (2008) examinan el impacto de la ayuda destinada a la educación en los avances en educación en 96 países de renta baja y media en el período 1970-2004, a través del empleo de modelos estáticos con efectos fijos y modelos dinámicos estimados con el método de momentos generalizado (MGM) y el método de mínimos cuadrados ordinarios en dos fases (2MCO). El análisis sugiere que la mayor ayuda educativa per cápita incrementa de una forma significativa la escolarización en la enseñanza Primaria, mientras que el incremento del gasto gubernamental nacional en educación no lo hace. Este resultado es consistente a cambios en el método de estimación, al uso de instrumentos para controlar la endogeneidad de la ayuda y al conjunto de variables de control incluidas en las estimaciones.

Por otro lado, Gymah-Brempong y Asiedu (2008) analizan si la ayuda externa desembolsada tiene un efecto significativo en la formación de capital humano (educación y salud) en 90 países en desarrollo en el período 1990-2004, mediante el uso de un modelo estático con efectos fijos y un panel de datos dinámico, que estiman con el sistema MGM. Encuentra que la ayuda educativa tiene un efecto positivo muy significativo en los avances de la educación en países en desarrollo. Hay evidencias de diferencias regionales del efecto de la ayuda en los avances de la educación. Asimismo, se observa que la ayuda agregada no está correlacionada de un modo significativo con los avances de la Educación Primaria.

Findley et al. (2009) examinan el efecto de la ayuda foránea en diversos sectores, entre los que se encuentra la educación, en 113 países, a través de la utilización de un modelo lineal jerárquico y de emparejamiento. El efecto estimado de la ayuda educativa en el promedio de años de escolarización es negativo y significativo desde un punto de vista estadístico, lo que sugeriría para un país receptor medio que la ayuda educativa no incrementa el logro educativo. Es posible que esta estimación sea el resultado de efectos selección en la asignación de la ayuda educativa. El efecto tratamiento promedio es negativo, pero es no significativo desde una perspectiva estadística, lo que conduce a la conclusión más favorable de que la ayuda educativa no tiene un efecto claro en el logro educativo en el corto plazo. Además, detectan que el logro educativo pasado no es un fuerte predictor de la ayuda educativa, por lo que es menos plausible que la correlación negativa que se encuentra entre la ayuda y el logro educativo sea causada por la selección.

Christensen, Homer y Nielson (2010) evalúan de una forma empírica el efecto de la ayuda educativa foránea comprometida en los avances de la educación en 109 países 
de renta baja y de renta media-baja, en el período 1975-2005, mediante el empleo de un modelo estático con efectos fijos (MCO) y un modelo curvilíneo de crecimiento latente. Los resultados sugieren que la ayuda a la Educación Primaria no está vinculada con las tasas de matrícula primaria de un modo significativo desde el punto de vista estadístico. No hay una evidencia real de que el incremento de la ayuda a la Educación Primaria haya mejorado las tasas de matrícula. Los hallazgos estadísticos indican que hay una pequeña relación significativa entre las dos variables en alguna regresión, lo que sugiere que el compromiso global de incrementar la escolarización primaria a través de la ayuda no es efectivo en conjunto, aunque es probable que haya historias de éxito individual para proyectos específicos.

Christensen, Homer y Nielson (2011) investigan los efectos de la ayuda comprometida destinada a Educación Primaria en la matrícula escolar de 100 países de renta baja y de renta media-baja, en el período 1995-2008, a través del uso de un modelo curvilíneo de crecimiento latente o modelo lineal jerárquico (MLJ). Los resultados sugieren que la ayuda a la Educación Primaria puede causar incrementos limitados de la matrícula escolar. Sin embargo, los hallazgos también indican que los efectos pueden no ser universales. Separar la ayuda bilateral de la multilateral produce resultados muy diferentes para los dos tipos de ayuda. Los hallazgos sugieren que la ayuda bilateral puede superar el problema de la selección adversa algo mejor que la ayuda multilateral. Los donantes bilaterales pueden condicionar su ayuda a la Educación Primaria y a la calidad del gobierno de los países receptores. No se puede afirmar de una forma definitiva que la efectividad de la ayuda a la educación se deriva de la distribución de la ayuda bilateral basada en la gobernanza. La existencia de evidencias consistentes desde una perspectiva estadística hace pensar en un vínculo amplio y general entre ayuda a la Educación Primaria y la matrícula en Educación Primaria.

Ziesemer (2011) examina la incidencia de la ayuda oficial al desarrollo en la tasa de alfabetización en 30 países de renta baja en el período 1985-2004, mediante la aplicación del método de mínimos cuadrados con efectos fijos. Los resultados muestran que la AOD y el gasto público en educación aumentan la alfabetización. La AOD es significativa desde un punto de vista estadístico y presenta el coeficiente estimado más alto, debido a que la ayuda está a menudo vinculada a la educación.

En un trabajo posterior, Ziesemer (2012) investiga el impacto de la ayuda oficial al desarrollo en la educación y en la salud en 65 países de renta baja, a través del uso del método de mínimos cuadrados con efectos fijos y un panel de datos dinámico, que estima con el sistema MGM. Los hallazgos indican que tanto el nivel como la tasa de crecimiento de la ayuda per cápita reducen las tasas de crecimiento del 
analfabetismo en las estimaciones de sistema MGM. La AOD significativa desde la perspectiva estadística y presenta un coeficiente estimado negativo. Las estimaciones tienen como resultado solo una muy débil endogeneidad para las variables de ayuda. Sin embargo, el uso de un inadecuado estimador MCO con frecuencia presenta resultados no significativos para la ayuda.

D'Aiglepierre y Wagner (2013) analizan la relación entre la ayuda a la educación y los avances en la educación de 101 países en desarrollo en el período 1999-2009, mediante la utilización de modelos estáticos con efectos fijos, que estiman con los métodos MCO y 2MCO. Los resultados son muy consistentes e indican que la ayuda a la Educación Primaria tiene un fuerte efecto en la matrícula en primaria y en la paridad de género. También se señala un impacto negativo sobre la tasa de repetición, mientras que se puede observar que no hay efecto ni sobre el cociente alumno-profesor ni sobre el porcentaje de profesores formados de Educación Primaria.

Birchler y Michaelowa (2013) examinan el efecto de la ayuda educativa desembolsada en la escolarización primaria y en la calidad de la educación en 110 países en desarrollo en el período 1996-2010, a través del empleo de un modelo estático con efectos fijos y un panel de datos dinámico, que estiman con el sistema MGM. Encuentran que el incremento de la financiación por parte de los donantes ha contribuido de una forma sustancial a un aumento en la matrícula. La ayuda educativa ha sido más relevante para mejorar la matrícula (cantidad de educación) que para incrementar el logro (calidad de educación). Hallan que un incremento de la ayuda educativa del $1 \%$ aumenta la tasa de crecimiento de la matrícula primaria en 0,06 puntos porcentuales. Este resultado es modesto en términos relativos, pero no desdeñable. El efecto positivo observado se dirige en especial a aquellas subcategorías que están relacionadas de un modo directo con la Educación Primaria. El efecto más consistente en la escolarización primaria se obtiene en la categoría instalaciones educativas y formación.

Además, hay evidencias de complementariedades entre la ayuda en Educación Primaria y la dirigida a la enseñanza Secundaria. La financiación de estos diferentes niveles educativos tiene un efecto que se refuerza de una forma mutua. También realizan un análisis comparativo cualitativo (ACC) de la calidad de la educación que muestra la importancia de una mezcla equilibrada entre los niveles Primario y Superior de la educación para la mejora de la calidad de la Educación Primaria.

Por último, Maïga (2014) investiga el impacto de la ayuda educativa foránea desembolsada en los resultados de igualdad de género en educación en 142 países en desarrollo, en el período 2002-2011, mediante la utilización del sistema MGM y el método MCO. Se encuentra que la ayuda educativa agregada desembolsada 
tiene un impacto no significativo sobre la paridad de género en todos los niveles de educación combinados y sobre la paridad de género en Educación Primaria. Su impacto sobre la Educación Secundaria y Terciaria es negativo y significativo. En el caso de la ayuda por subsectores específicos, los coeficientes son positivos y no significativos para el índice de paridad de género (IPG) en la matrícula en Educación Primaria y Secundaria, y negativo y no significativo para el IPG en educación terciaria. Estos resultados no son consistentes con la especificación usada o con el método de estimación. No parece importar ni la heterogeneidad en el tipo de receptor de la ayuda ni la heterogeneidad en los flujos de ayuda.

\section{CONCLUSIONES}

En un elevado número de estudios, la ayuda educativa tiene un impacto positivo y significativo en variables educativas flujo (tasa de matrícula), stock (tasa de alfabetización y tasas de finalización). No obstante, también hay algunos estudios (menos numerosos) en los que dicho impacto es negativo y / o no significativo. Es decir, que los resultados en relación con dicho vínculo no son del todo concluyentes. La ausencia de resultados consistentes y las limitaciones metodológicas no resueltas evitan el consenso en este tema particular.

En este trabajo solo se han revisado estudios econométricos. Dicha revisión es la aportación principal al tema objeto de estudio. Una línea de investigación que queda abierta sería la realización de análisis en los que se utilicen otros tipos de estudios que puedan ser relevantes para investigar los vínculos entre la ayuda educativa y los avances en la educación y, de esta manera, poder lograr resultados más concluyentes.

\section{BIBLIOGRAFÍA}

Anwar, M. y Aman S. (2010). Aid effectiveness in education sector of Pakistan. HWWI - Hamburg Institute of International Economics, Research Paper, 16p

Arvin, B.M. y Lew, B. (2009). Foreign aid and child educational attainment in developing countries. En: International Journal of Education Economics and Development, Vol.1, No. 2, p. 166-178.

Asiedu, E y Nandwa, B. (2007). The impact of foreign aid in education growth. How relevant is the heterogeneity of aid flows and the heterogeneity of aid recipients? En: Review of World Economics, Vol.143, No. 4, p. 631-649.

Asiedu, E. (2014). Does foreign aid in education promote economic growth? Evidence from Sub-Saharan Africa. En: Journal of African Development, Vol.16, No. 1, spring, p. 37-59.

Birchler, K y Michaelowa, K. (2013). Making aid work for education in developing countries. An analysis of aid effectiveness for primary education coverage and quality. WIDER- World Institute for Development Economics Research, Working Paper, No. 2013/21, 27p. 
Clemens, M.A.; Radelet, S. y Bhavnani, R. (2004). Counting chickens when they hatch: the short term effect of aid on growth. CGD- Center for Global Development, Working Paper, No. 44, Washington, DC., 63p.

Christensen, Z.; Homer, D. y Nielson, D. (2010). We don't need no education. The effects of education-specific foreign aid on school enrollment in low-income countries. Prepared for presentation at the Conference Aid Transparency, $25 \mathrm{p}$.

Christensen, Z.; Homer, D. y Nielson, D. (2011). Dodging adverse selection: How donor type and governance condition aid's effects on school enrollment? En: World Development, Vol. 39, No. 11, p. 2044-2053.

D'Aiglepierre, R. y Wagner, L. (2013). Aid and universal primary education. En: Economics of Education Review, Vol.37, diciembre, p. 95-112.

Dreher, A; Gehring, K. y Klasen, S. (2013). Gesture politics of real commitment? Gender inequality and the allocation of aid. WIDER- World Institute for Development Economics Research. Working Paper, No. 2013/079, 37p.

Dreher, A.; Nunnenkamp, P. y Thiele, R. (2008). Does aid for education educate children? Evidence from panel data. En: World Bank Economic Review, Vol. 22, No. 2, p. 291-314.

Fielding, D.; Mc.Gillivray, M. y Torres, S. (2006). A wider approach to aid effectiveness. Correlated impacts on health, wealth, fertility and education. Research Paper $n^{\circ}$ 2006/23 UNU-WIDER-United Nations University - World Institute for Development Economics Research, 16p.

Findley, M.; Hawkins, D.; Nielsen, R.; Nielson, D. y Wilson, S. (2009). To empower or impoverish? The sector - by sector effectiveness of foreign aid. Paper presented at the Aid Data Oxford Conference, marzo 22-25, 2010, 53p.

Gymah-Brempong, K. y Asiedu, E. (2008). Aid and human capital formation: some evidence. Paper to be presented at the African Development Bank Conference on Globalization, Institution and Economic Development in Africa, 27p.

Kasuga, H. (2008). Aid allocation across sector: does aid fit well with recipients' development priorities? RIETI-Research Institute of Economy, Trade and Industry, Discussion Paper Series, No. 08-E-039, 34p.

Kleemann, L.; Nunnenkamp, P y Thiele, R. (2014). Gender Inequality, female leadership and aid allocation. A panel analysis of aid for education. WIDER-World Institute for Development Economics Research, Working Paper, No. 2014/010, 28p.

Maïga, E.W.H. (2014). Does foreign aid in education foster gender equality in developing countries? WIDER-World Institute for Development Economics Research, Working Paper, No. 2014/048, $22 \mathrm{p}$.

Michaelowa, K. (2004). Aid effectiveness reconsidered - Panel data evidence for the education sector. HWWA-Hamburg Institute of International Economics, Discussion Paper, No. 264, 21p.

Michaelowa, K. y Weber, A (2007a). Aid effectiveness in the education sector - a dynamic panel analysis, p. 357-385. En: Lahiri, S. (ed.): Book Series: Frontiers of Economics and Globalization, Volume 1 - Theory and Practice of Foreign Aid. Editorial Emerald, 438p. 
Michaelowa, K. y Weber, A. (2007b). Aid effectiveness in primary, secondary and tertiary education. Background Paper prepared for the Education for All Global Monitoring Report 2008. Education for All by 2015: will we make it?, 22p.

Moe, T. L. (2008). An empirical investigation of relationships between official development assistance (ODA) and human and educational development. En: International Journal of Social Economics, Vol. 35, No. 3, p. 202-221.

Neira, I. y Portela, M. (2003). Cooperación y desarrollo: el papel de la educación en el desarrollo latinoamericano, p. 131-144. En: San Segundo, M.J. y Zorrilla, R. (eds): Actas de las XII Jornadas de la AEDE, Ed. AEDE-Asociación de Economía de la Educación, IEF-Instituto de Estudios Fiscales y Universidad Carlos III de Madrid.

Neira, I. y Portela, M. (2004). La situación latinoamericana a comienzos del siglo XXI. En: Archivos Analíticos de Política Educativa, Vol.12, No. 15, 23p.

Thiele, R.; Nunnenkamp, P. y Dreher, A. (2007). Do donors target aid in line with the Millenium Development Goals? A sector perspective of aid allocation. En: Review of World Economics, Vol. 143, No. 4, p. 596-630.

Van de Sijpe, N. (2010). Is foreign aid fungible? Evidence from the education and health sectors. Working Paper, No. 688/2010, Universiteit Gent, 56p.

Wolf, S. (2007). Does aid improve public service delivery? En: Review of World Economics, Vol. 143, No. 4, p. 650-672.

Yogo, U.T. y Mallaye, D. (2014): How aid helps achieving MDGs in Africa: the case of primary education. MPRA-Munich Personal RePEc Archive paper, No. 602012, 25p.

Ziesemer, T. (2011). Growth with endogenous migration hump and the multiple, dynamically interacting effects of aid in poor developing countries. En: Applied Economics, Vol. 43, No. 30, p. 4865-4878.

Ziesemer, T. (2012). The impact of development aid on education and health: survey and new evidence from dynamic models. Working Paper, No. 2012/57 UNUMERIT- United Nations University-Maastricht Economic and social Research Institute on Innovation and Technology, $34 \mathrm{p}$. 
